\title{
Le comportement alimentaire du cabri créole élevé en stabulation libre de la naissance au sevrage
}

\author{
F. LEVY * et Gisèle ALEXANDRE \\ I.N.R.A., Station de Zootechnie, C.R.A.A.G. \\ F 97170 Petit-Bourg, Guadeloupe
}

\begin{abstract}
Résumé
Le comportement de tétée et le comportement vis-à-vis d'aliments solides (fourrage et aliment concentré) ont été observés chez 2 groupes de chevreaux créoles de la naissance au sevrage (12 ${ }^{\mathrm{e}}$ semaine). Dans le lot BN (12 chevreaux) les mères étaient nourries avec du fourrage vert ad libitum (Digitaria decumbens) alors que dans le lot HN (14 chevreaux) les mères recevaient en plus de l'aliment concentré $(0,5 \mathrm{~kg} /$ tête/jour $)$. Les chevreaux des 2 groupes avaient libre accès à l'alimentation des mères et à partir de la $3^{\mathrm{e}}$ semaine, de l'aliment concentré de sevrage était distribué dans une mangeoire accessible aux seuls jeunes. Des observations étaient effectuées une fois par semaine de $6 \mathrm{~h}$ à $12 \mathrm{~h}$ et à la $5^{\circ}$ semaine nous avons relevé le nombre de tétées pendant un nycthémère.
\end{abstract}

La fréquence des tétées et des tentatives ainsi que la durée des tétées ne diffèrent pas entre les 2 lots. Jusqu'à la $5^{\mathrm{e}}$ semaine cette fréquence est élevée (13 tétées/chevreau/ $6 \mathrm{~h})$ puis décroît significativement à la $6^{\circ}$ semaine $(6$ tétées/chevreau/ $6 \mathrm{~h})$. La durée ne varie au cours de l'allaitement que pour le lot $\mathrm{BN}\left(26 \mathrm{~s}\right.$ jusqu'à la $4^{*}$ semaine vs $18 \mathrm{~s}$ de la $5^{\mathrm{e}}$ à la $12^{\mathrm{e}}$ semaine). Le nombre de tentatives évolue parallèlement à la fréquence de tétées. Celle-ci diffère peu entre les chevreaux simples et doubles. Pendant le nycthémère, le nombre de tétées par tranches de 6 heures est significativement plus faible entre 0 et $6 \mathrm{~h}$ mais ne varie pas au cours du reste de la journée.

Le démarrage de l'ingestion de fourrage vert a lieu sensiblement en même temps dans les 2 lots $\left(3^{\circ}\right.$ vs $4^{\mathrm{e}}$ semaine ; lot $\mathrm{HN}$ et $\mathrm{BN}$ respectivement). En revanche, l'ingestion d'aliment concentré de sevrage apparaît nettement plus précocement chez les jeunes dont les mères reçoivent une alimentation complémentaire ( $3^{\mathrm{e}}$ vs $6^{\mathrm{e}}$ semaine; lot $\mathrm{HN}$ et $\mathrm{BN}$ respectivement).

Ces observations révèlent une période de 5 semaines de dépendance étroite vis-à-vis du lait maternel quel que soit le niveau d'alimentation des mères; elles mettent également en évidence un phénomène de transmission par imitation du comportement alimentaire par la mère qui peut avoir des implications pratiques importantes.

Mots clés : chevreau créole, comportement de tétée, comportement alimentaire, apprentissage alimentaire.

(*) Adresse actuelle : I.N.R.A., Centre de Recherches de Tours, Station de Physiologie de la Reproduction, Nouzilly, 37380 Monnaie. 


\section{Introduction}

Aux Antilles françaises, les chèvres locales ou cabris créoles sont exclusivement élevées en système allaitant pour la production de viande. De par son intérêt socioéconomique et ses possibilités d'exploitation en milieu tropical, cet élevage caprin est d'une très grande importance. En effet, la viande de cabri fait l'objet d'une grande demande de la part de la population locale et son prix est élevé. A l'heure actuelle, l'élevage de type familial ( 1 à 5 têtes par famille) ne répondant pas aux besoins, il est appelé à se développer par la création de plus grandes unités (80 à 100 têtes) mais toujours à caractère familial. Son développement repose toutefois sur une meilleure connaissance biologique de l'animal permettant la mise au point de techniques d'élevage adéquates. En effet, les poids et les âges d'abattage sont très médiocres (18 kg à 24 mois) ; jusqu'au sevrage, le taux de mortalité des chevreaux peut s'élever à $26 \mathrm{p} .100$ et le gain de poids journalier ne dépasse pas $62 \mathrm{~g}$ (CogNIE et al., 1971). Ces quelques donnćes montrent que l'un des principaux problèmes concerne l'élevage des jeunes et notamment l'alimentation pendant et après la période d'allaitement. Avant le sevrage, deux solutions sont envisageables : augmenter la production laitière des mères en améliorant l'apport alimentaire azoté (ALEXANDRli, 1983) ou fournir aux jeunes des aliments solides ingestibles énergétiques et azolés ainsi que Brown (1964), Spedding \& CharLet (1967) le préconisent pour les agneaux sevrés précocement. Toutefois le choix de stratégies alimentaires pendant l'allaitement et la préparation au sevrage nécessitent la connaissance du comportement alimentaire du chevreau allaité par la mère.

Les données étant inexistantes en la matière chez le cabri créole, il nous est apparu nécessaire d'entreprendre, en premier lieu, une étude descriptive du comportement alimentaire jusqu'au sevrage (comportement de tétée et comportement visà-vis d'aliments solides) qui nous permettrait de connaître :

- la période d'allaitement strict et le passage progressif à l'alimentation solide,

- l'influence du niveau et du mode d'alimentation des mères.

\section{I. Matériel et méthodes}

L'étude s'est déroulée à la Station de Recherches Zootechniques du Centre de Recherches Agronomiques des Antilles et de la Guyane situé en Guadeloupe (milieu tropical humide). De février à avril 1983 (saison sèche), nous avons observé de la naissance au sevrage ( 84 jours) des chevreaux créoles élevés sous la mère en stabulation libre. Pendant ces observations la durée de la période claire du nycthémère était de 12 heures et le jour se levait entre 6 h et 6 h 15 .

\section{A. Lots expérimentaux}

La reproduction du troupeau est synchronisée par regroupement des ovulations (pose d'éponges vaginales contenant un progestagène) et des mise-bas (injection de dexaméthasone, glucocorticoïde de synthèse). Ainsi les naissances sont-elles concentrées sur une période de $48 \mathrm{~h}$. 
Juste après la parturition, les chèvres et leurs jeunes ont été répartis, en tenant compte du poids de la mère et de la taille de la portée, en deux lots expérimentaux selon le niveau d'alimentation :

- Bas niveau alimentaire (BN) : 6 mères de simples et 3 mères de doubles reçoivent une ration de fourrage vert ad libitum (Pangola «digitaria decumbens»).

- Haut niveau alimentaire $(\mathrm{HN})$ : 5 mères de simples, 3 mères de doubles et 1 mère de triples reçoivent en plus du Pangola ad libitum, $0,5 \mathrm{~kg} / \mathrm{tête} / \mathrm{jour}$ d'un aliment concentré granulé, composé de maïs, de son et de tourteau de soja, de $4,5 \mathrm{~mm}$ de diamètre.

Les quantités de matière sèche et d'énergie ingérées correspondant aux deux niveaux alimentaires sont identiques à celles rapportées par ALEXANDrE (1983). En outre, on doit noter le faible taux de substitution concentré/Pangola $(0,33$ à 0,53 ; AlexandRe, 1983).

Ces 2 groupes sont élevés dans 2 parcs de $4 \mathrm{~m}$ sur $3 \mathrm{~m}$ séparés par une barrière à claire-voie. La distribution de l'aliment concentré aux chèvres avait lieu à $8 \mathrm{~h}$ et celle de Pangola à $9 \mathrm{~h}$ et $14 \mathrm{~h}$ pour les 2 lots. Dès la naissance, les chevreaux avaient libre accès à l'alimentation des mères. Dans chaque parc, une mangeoire accessible seulement aux chevreaux avait été aménagée. A partir de la $3^{\circ}$ semaine et jusqu'au sevrage, de l'aliment concentré granulé "sevrage » de même composition mais de taille de granulation inférieure à celui de la mère $(2,5 \mathrm{~mm}$ de diamètre $) \mathrm{y}$ était distribué tous les matins à $6 \mathrm{~h}$.

\section{B. Mesures comportementales}

Une fois par semaine, chaque lot était observé de $6 \mathrm{~h}$ à $12 \mathrm{~h}$ par un des deux expérimentateurs. Cette période a été choisie parce qu'elle encadrait celle de la distribution de Pangola et de concentré.

Dès la naissance, le nombre et la durée des tétées ainsi que le nombre de tentatives infructueuses de chaque chevreau étaient enregistrés au magnétophone. Deux tétées consécutives séparées de moins de $60 \mathrm{~s}$ étaient assimilées à une seule tétée. Nous avons appelé tentative de tétée toute séquence comprenant un passage au poitrail de la mère et/ou une position d'allaitement suivie ou non d'une tétée d'une durée inférieure ou égale à 2 secondes.

A partir de la $3^{\mathrm{e}}$ semaine, nous relevions, en outre, par intervalle de 10 minutes pour chaque chevreau l'ingestion de fourrage et d'aliment « sevrage». L'animal n'était pris en compte que s'il avait été vu s'alimenter au moins une fois dans l'intervalle des 10 minutes. Afin de comparer ces 2 activités alimentaires, nous avons pris en compte le temps pendant lequel l'aliment (fourrage ou concentré) était présent. Nous avons ainsi défini un taux d'activité alimentaire :

Nombre de périodes de $10 \mathrm{mn}$ pendant lesquelles de l'aliment est ingéré

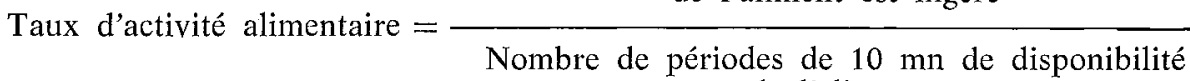
de l'aliment 
Ainsi dans les cas extrêmes, le taux prend-il la valeur 0 quand le chevreau ne vient jamais s'alimenter ou la valeur 1 quand le chevreau s'alimente pendant tout le temps où l'aliment est disponible. L'ingestion d'aliment concentré par les mères du lot $\mathrm{HN}$ ne durant que $15 \mathrm{mn}$, nous n'avons pas pris en compte l'activité alimentaire des jeunes pendant cette période.

Afin d'évaluer la fréquence nycthémérale d'allaitement nous avons relevé, à la $5^{\circ}$ semaine pour des raisons pratiques, le nombre de tétées par chevreau pendant 24 heures consécutives.

\section{Analyse statistique}

Nous avons utilisé le test statistique de Mann-Whitney pour la comparaison inter-lots et le test de Wilcoxon pour la comparaison intra-lots (SiEgeL, 1956). Le seuil de signification est fixé à 0,05 .

\section{Résultats}

\section{A. Evolution du comportement de tétée}

A la $5^{\prime \prime}$ semaine, le nombre moyen de tétées par tranches de 6 h est significativement plus faible entre 0 et $6 \mathrm{~h}$ mais ne varie pas significativement au cours du reste de la journée et ceci dans les 2 lots (tabl. 1). La fréquence d'allaitement pendant la période diurne $(6 \mathrm{~h}-18 \mathrm{~h})$ est significativement supérieure à celle observée

\section{TABLEAU 1}

Fréquence d'allaitement durant un nycthémère chez le cabri créole âgí de 5 scmaines.

Suckling frequency during a 24 h period in 5 week old Creole kids.

\begin{tabular}{|c|c|c|c|c|}
\hline $\begin{array}{l}\text { Nombre moyen de tétées par che- } \\
\text { vreau } \pm \text { s.c.m. } \ldots \ldots \ldots \ldots \ldots \ldots \\
\text { Mean number of suckings per } \\
\text { kid } \pm \text { s.e.m. }\end{array}$ & $0-6$ h & $6-12 \mathrm{~h}$ & $12-18 \mathrm{~h}$ & $18-24 \mathrm{~h}$ \\
\hline $\begin{array}{l}\text { Lot ì haut niveau dalimentation } \\
\quad(\mathrm{n}=14) \mathrm{HN} \ldots \ldots \ldots \ldots \ldots \ldots \\
\text { High feeding level group } H L\end{array}$ & $\begin{array}{c}6,8 \pm 0,5 \\
\text { a }\end{array}$ & $\begin{array}{c}11,8 \pm 1,3 \\
b\end{array}$ & $11,5 \pm 1,3$ & $11 \pm 1,1$ \\
\hline $\begin{array}{l}\text { Lot à bas niveau d'alimentation } \\
(\mathrm{n}=12) \mathrm{BN} \ldots \ldots \ldots \ldots \ldots \ldots \\
\text { Low feeding level group LL }\end{array}$ & $\begin{array}{c}8,4 \pm 0,8 \\
\mathrm{a}\end{array}$ & $\begin{array}{c}10,5 \pm 3,9 \\
b\end{array}$ & $9,7 \pm 1$ & $\begin{array}{c}10,1 \pm 0,5 \\
b\end{array}$ \\
\hline $\begin{array}{l}\text { (a, b) I-es valeurs affectées de lettres } \\
\text { Values with different letter }\end{array}$ & $\begin{array}{l}\text { différentes } \\
\text { differ. }\end{array}$ & èrent entre & & \\
\hline
\end{tabular}



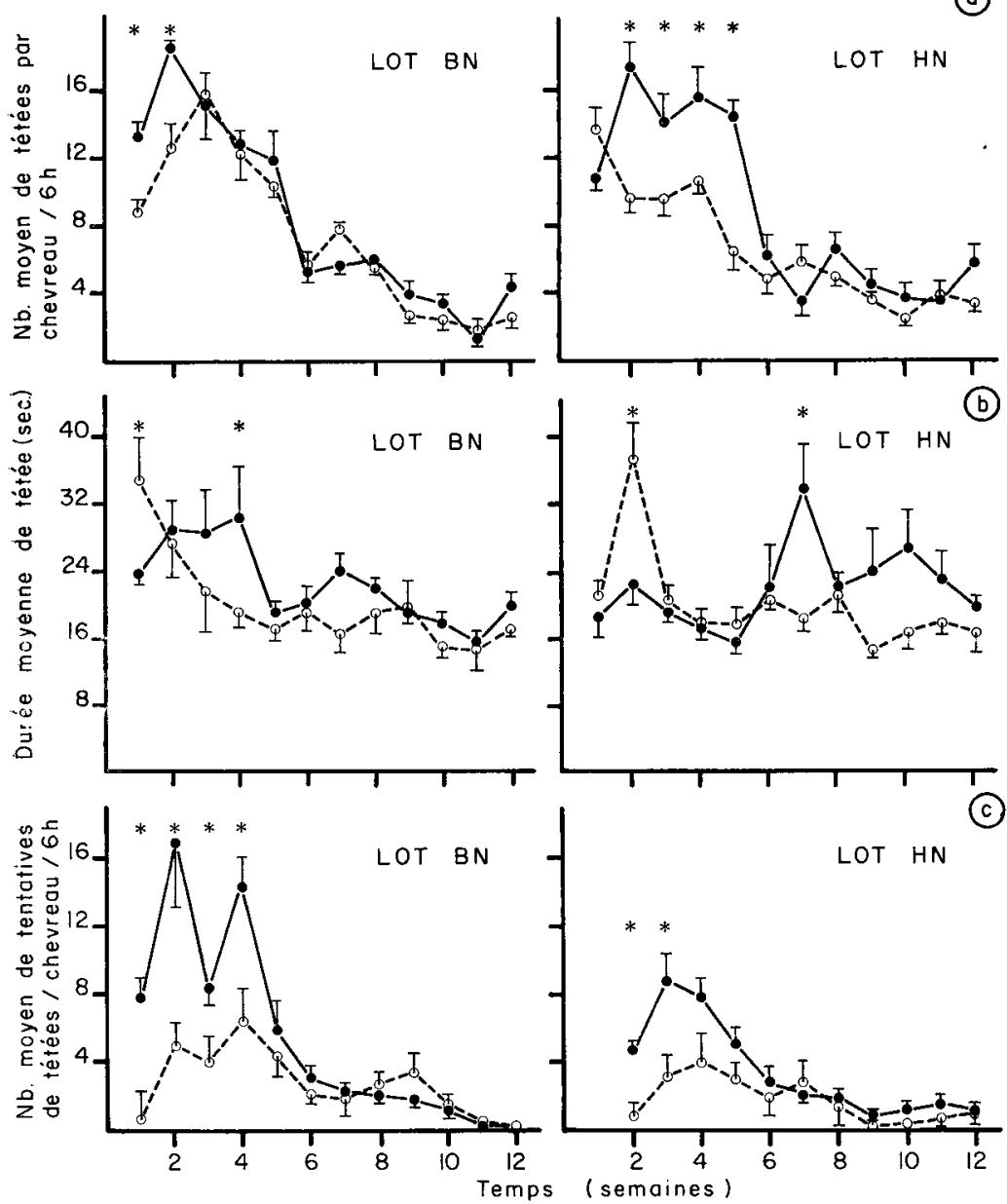

FIG. 1

Evolution des paramètres du comportement de tétée observé hebdomadairement pendant 6 heures de la naissance au sevrage chez le cabri créole élevé en stabulation libre.

Development of sucking behaviour observed weekly during a 6 hour period from birth to weaning in the Creole kid kept in loose housing.

FIG. 1 a

Fréquence des tétées.

Sucking frequency.

\section{FIG. $1 \mathrm{~b}$}

Durée moyenne des tétées. Mean length of suckings.

Fig. $1 \mathrm{c}$

Fréquence des tentatives de tétées.

Frequency of sucking attempts.

Lot BN : Les mères reçoivent du fourrage (Pangola) ad libitum.

LL group : Dams fed with forage (Pangola) ad libitum.

Lot HN : Les mères reçoivent du fourrage ad libitum et $0,5 \mathrm{~kg} / \mathrm{tête} /$ jour d'aliment concentré.

HL group : Dams fed with forage ad libitum and $0.5 \mathrm{~kg} /$ head/day of pellets.

-1 : Chevreaux simples : $\mathrm{N}=6$ pour le lot $\mathrm{BN}$. $\mathrm{N}=5$ pour le lot $\mathrm{HN}$. Simple kids : $N=6$ in the LL group. $N=5$ in the $H L$ group.

O-- : Chevreaux doubles : $\mathrm{N}=6$ pour le lot $\mathrm{BN} . \mathrm{N}=9$ pour le lot $\mathrm{HN}$ (le triplet inclus).

: Twin kids : $N=6$ in the $L L$ group. $N=9$ in the $H L$ group (triplet included).

: Différence significative entre chevreaux simples et doubles, $\mathrm{p}<0.05$.

Significative difference between simple and twin kids, $p<0.05$. 

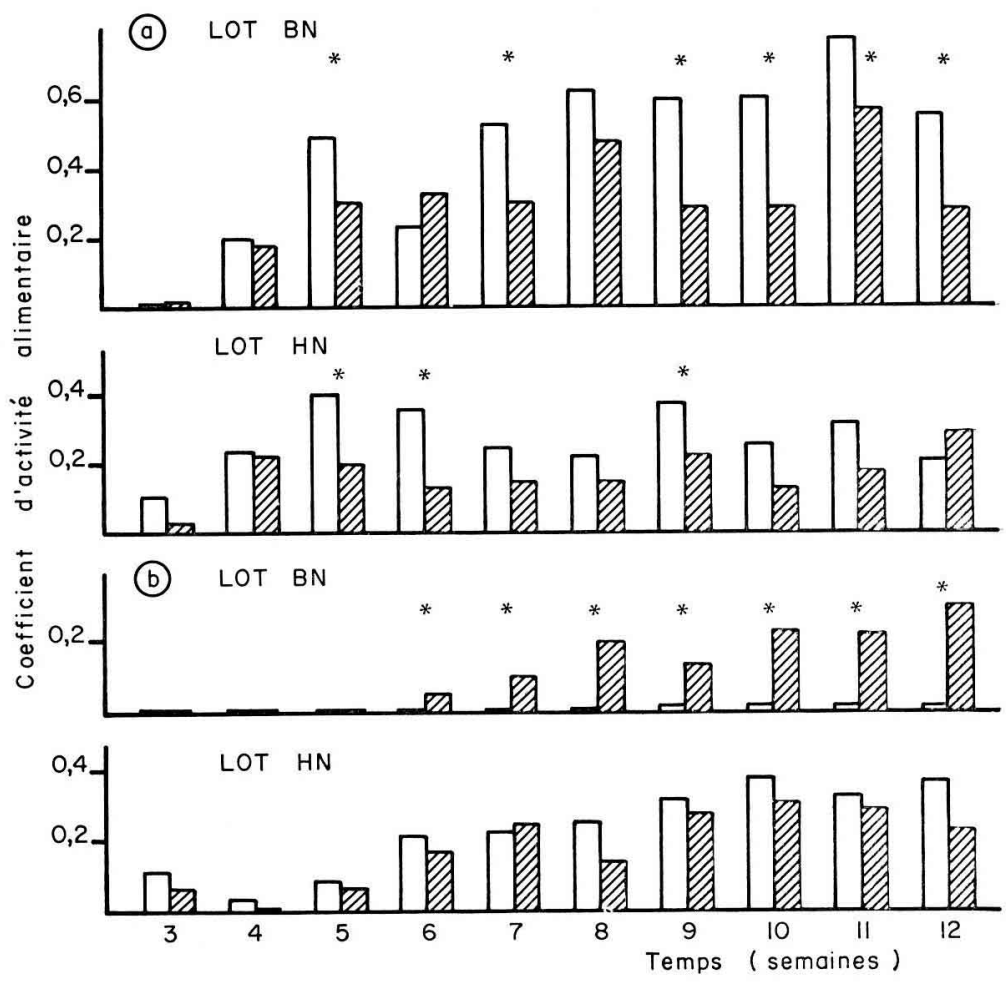

FIG. 2

Evolution du comportement alimentaire observé hebdomadairement pendant 6 heures de la $3^{\circ}$ à la $12^{\prime \prime}$ semaine chez le cabri créole élevé en stabulation libre.

Development of feeding behaviour observed weekly during a 6 hour period in the Creole kid kept in loose housing.

FIG. 2 a

Evolution du comportement alimentaire vis-à-vis du fourrage (Pangola).

Development of feeding behaviour relative to forage (Pangola).

FIG. $2 \mathrm{~b}$

Evolution $d u$ comportement alimentaire vis-à-vis d'un aliment concentré de sevrage. Development of feeding behaviour relative to weaning pellets.

Lot BN : cf. fig. 1.

\section{LL group.}

Lot HN : cf. fig. 1.

$H L$ group.

एथa chevreaux simples. simple kids.

$\square$ chevreaux doubles. twin kids.

* différence significative entre chevreaux simples et doubles $p<0,05$. significative difference between simple and twin kids $p<0.05$. 
pendant la période nocturne dans le lot $\mathrm{HN}(11,7 \pm 1$ vs $8,7 \pm 1)$. Mais cette différence n'existe pas dans le lot $\mathrm{BN}(10,5 \pm 1$ vs $9,5 \pm 0,5)$. La comparaison inter-lots montre que pendant la période diurne, la fréquence d'allaitement du lot HN est supérieure à celle du lot $\mathrm{BN}(11,7 \pm 1$ vs $10,5 \pm 1)$. La période retenue pour les observations hebdomadaires ( $6 \mathrm{~h}$ à 12 h) représente $30 \mathrm{p} .100$ des tétées pour le lot HN (172 sur 574); cette proportion diffère de celle correspondant à l'hypothèse d'une absence d'évolution pendant le nycthémère (172 sur 574 vs 143 sur 574 ; $\mathrm{P}<0,05)$. Par contre le lot $\mathrm{BN}$ ne présente pas une telle variation (28 p. 100 des tétées entre 6 h et 12 h; 136 sur 486 vs 121 sur 486).

Quelle que soit la semaine considérée, le nombre de tétées observées par chevreau pendant $6 \mathrm{~h}$ ne diffère pas significativement en fonction du niveau nutritionel (fig. 1 a). La fréquence des tétées évolue avec le temps. Jusqu'à la $5^{\mathrm{e}}$ semaine elle est élevée dans les 2 lots (13 tétées par chevreau observées par $6 \mathrm{~h}$ ). Entre $1 \mathrm{a} 5^{\mathrm{e}}$ et $6^{\text {e }}$ semaine elle diminue significativement dans les 2 lots (12 tétées par chevreau à la $5^{\prime \prime}$ semaine vs 6 tétées par chevreau à la $6^{\prime \prime}$ semaine). Ultérieurement jusqu'au sevrage la moyenne n'est plus que de 4 tétées par chevreau.

La fréquence d'allaitement des chevreaux simples est significativement supérieure à celles des doubles de la $2^{\circ}$ à la $5^{\prime \prime}$ semaine pour le lot $\mathrm{HN}$ et pendant les 2 premières semaines pour le lot $\mathrm{BN}$.

Il n'existe aucune différence significative de la durée moyenne de tétées par chevreau entre les 2 lots (fig. $1 \mathrm{~b}$ ). Cette durée n'évolue au cours de l'allaitement que pour le lot $\mathrm{BN}$ (26 sec jusqu'à la $4^{\circ}$ semaine vs $18 \mathrm{sec}$ de la $5^{\mathrm{e}}$ à la $12^{\circ}$ semaine ; valeurs moyennes pour l'ensemble du lot). Enfin il n'apparaît que rarement de différence significative de durée de tétée entre les simples et les doubles : à la $2^{\circ}$ et $7^{\circ}$ semaine pour le lot $\mathrm{HN}$ et à la $1^{\mathrm{ro}}$ et $4^{\circ}$ semaine pour le lot $\mathrm{BN}$.

Le nombre moyen de tentatives observées par chevreau pendant $6 \mathrm{~h}$ ne diffère entre les 2 groupes qu'à la $2^{\prime \prime}$ et à la $4^{\circ}$ semaine $(3 \pm 0,6$ vs $11 \pm 2 ; 6 \pm 1$ vs $10 \pm 2$; lot $\mathrm{HN}$ versus lot $\mathrm{BN}$ respectivement $\mathrm{P}<0,01$; fig. $1 \mathrm{c})$. Cette valeur évolue parallèlement à celle du nombre des tétées : élevée pendant les 4 premières semaines ( 7 en moyenne pour les 2 lots) elle diminue significativement dès la $5^{\circ}$ semaine ( $8 \pm 1$ vs $5 \pm 1$; moyennes des 2 lots de la $4^{\mathrm{e}}$ vs $5^{\mathrm{e}}$ semaine) pour s'annuler à la $12^{\mathrm{e}}$ semaine $(0,5$ tétée par chevreau). Quel que soit le lot, les tentatives de tétées sont plus nombreuses chez les simples jusqu'à la $4^{\mathrm{c}}$ semaine puis cette différence s'annule.

\section{B. Evolution du comportement alimentaire vis-à-vis du fourrage vert}

Les résultats sont illustrés par la figure 2 a.

Dans le lot à bas niveau alimentaire, le Pangola est ingéré par les jeunes à la 4 " semaine. Les doubles passent significativement plus de temps à l'auge que les simples, mais essentiellement à partir de la $9^{\prime \prime}$ semaine.

Le démarrage de l'ingestion de fourrage vert du lot à haut niveau alimentaire a lieu dès la $3^{\circ}$ semaine. Mises à part 3 observations, les doubles ont un taux d'activité alimentaire pour le Pangola équivalent à celui des simples. 
Enfin dans 7 observations sur 12, les chevreaux du lot $B N$ totalisent un temps à l'auge contenant le Pangola significativement supérieur à celui du lot HN.

\section{Evolution du comportement alimentaire vis-à-vis de l'aliment concentré de sevrage}

Les résultats sont représentés sur la figure $2 \mathrm{~b}$.

Dans le lot à bas niveau d'alimentation, nous n'avons jamais observé de chevreau s'alimenter dans l'auge contenant de l'aliment de sevrage jusqu'à la 5 " semaine. A partir de la $6^{\circ}$ semaine, seuls les chevreaux simples en ingèrent. A l'exception d'un chevreau, les doubles ne se sont jamais alimentés dans cette auge jusqu'au sevrage.

En revanche, dans le lot à haut niveau alimentaire, 12 jeunes sur 14 se sont alimentés à l'auge dès la $3^{\prime \prime}$ semaine et à partir de la 6" semaine le taux d'activité alimentaire est égal à 0,21 alors que pour le lot $\mathrm{BN}$ il est de 0,05 . Pour chaque observation hebdomadaire, il n'y a jamais de différence significative entre les simples et les doubles.

Contrairement à ce que nous aurions pu attendre, le démarrage de la consommation de l'aliment concentré de sevrage est nettement plus précoce chez les jeunes dont les mères reçoivent une alimentation complémentaire.

\section{Discussion - Conclusion}

Nos résultats indiquent que chez le cabri créole élevé en stabulation libre, les tétées sont réparties uniformément entre $6 \mathrm{~h}$ et $24 \mathrm{~h}$ avec une baisse notable entre 0 et $6 \mathrm{~h}$, c'est-à-dire pendant la $2^{\circ}$ moitié de la période nocturne. Le niveau d'alimentation des mères et par conséquent la production laitière (ALEXANDRE, 1983) ne semble pas avoir d'influence sur cette répartition. Toutefois pour le lot à haut niveau d'alimentation nous notons un pic de tétées entre $6 \mathrm{~h}$ et $12 \mathrm{~h}$ qui s'expliquerait par les perturbations occasionnées par l'apport d'aliment concentré aux mères. Ce mode de répartition nycthémérale des tétées se rapproche de celui observé chez les Ovins élevés en bergerie où l'intervalle entre tétées de nuit est sensiblement équivalent à celui de jour (RicondeAu et al., 1960). Ce résultat se retrouve chez des Bovins élevés en stabulation libre (Sommerville \& Lowman, 1979). Toutefois des observations réalisées au pâturage chez cette espèce montrent que les tétées ont lieu principalement au lever du soleil (Le Neindre \& Petit, 1975). Ces différents schémas de distribution nycthémérale des fréquences de tétées seraient donc induits par des différences de conduite de troupeau plutôt que par des différences inter-spécifiques. Ainsi la période d'observation de 6 heures choisie au cours du nycthémère représentant environ le quart des tétées semble être représentative du comportement d'allaitement du cabri créole élevé en stabulation libre.

L'évolution de la fréquence de tétées jusqu'au sevrage peut se décomposer en deux phases. Jusqu'à la $5^{\mathbf{e}}$ semaine, elle est élevée, traduisant une dépendance étroite vis-à-vis du lait maternel. A partir de la $6^{\prime \prime}$ semaine les tétées deviennent de moins en moins fréquentes. Parallèlement à cette évolution le nombre de tentatives de tétées, 
traduisant en partie une recherche alimentaire, chute de façon importante entre la $4^{\mathrm{e}}$ et la $6^{\circ}$ semaine pour s'annuler juste avant le sevrage. Une évolution similaire a été rapportée chez des agneaux élevés en bergerie (RicondeAu et al., 1960) alors qu'au pâturage un nombre de tétées élevé ne s'observe que pendant les 2 à 3 premières semaines (EWBANK, 1967 ; Flechter, 1971). A âge et milieu d'élevage équivalents, les fréquences rapportées dans la littérature sont moindres que celles des chevreaux créoles. La durée moyenne de tétée du chevreau créole reste globalement constante jusqu'au sevrage alors que chez les Ovins elle diminue sensiblement après le premier mois de vie (EWBANK, 1967 ; RicordeAu et al., 1960).

Quel que soit l'âge du jeune, la différence de production laitière (mesurée hebdomadairement par la méthode ocytocinique, cf. AlexANDre, $1983 ; 875 \mathrm{~g} / \mathrm{j} \pm 50$ vs $506 \mathrm{~g} / \mathrm{j} \pm 60$, valeurs moyennes des lots sur les 12 semaines) consécutive à celle de niveau d'alimentation n'a pas de répercussion sur le nombre d'essais de tétées (excepté à la $2^{\circ}$ et $4^{\mathrm{e}}$ semaine) ainsi que sur leur fréquence et leur durée. En revanche, en début de lactation le comportement des chevreaux simples vis-à-vis de ces 3 paramètres est différent de celui des doubles. Cette différence s'estompe après la $5^{\text {e }}$ semaine. Chez les Ovins en bergerie, Ricordeau et al. (1960) n'ont pas montré de différence de fréquence de tétées entre les simples et les doubles alors que EWBANK (1967) observe au pâturage une fréquence supérieure chez les doubles durant les 3 premières semaines. Enfin chez la vache en plein air, les jumeaux tètent aussi fréquemment que les veaux simples (LE NeINDre, 1984).

Alors que les tétées diminuent très sensiblement après la $5^{\circ}$ semaine, l'ingestion conséquente de fourrage apparaît autour de cette même période, le lot à haut niveau d'alimentation étant plus précoce, ce qui est probablement dû à une croissance plus rapide. Une telle évolution du comportement alimentaire a été souvent rapportée chez l'agneau (Doney \& Peart, 1976 ; Langlands, 1977 ; Penning \& GibB, 1979) ainsi que chez le veau (Mathieu \& Wegat-Litre, 1961 ; Baker et al., 1976) entre la $4^{\mathrm{e}}$ et la $6^{\mathrm{e}}$ semaine et chez le chevreau à la $4^{\mathrm{e}}$ semaine (Economides et al., 1982). Cette phase de changement progressif de comportement correspondrait pour le jeune à la transition du stade de préruminant où le lait est à la base de son alimentation au stade intermédiaire entre préruminant et ruminant où la digestion d'aliments solides est rendue possible par le développement de son rumen qui s'accélérerait dès la $3^{\text {e }}$ semaine chez le chevreau (Church, 1976 ; Gihad \& Morad, 1977) et chez l'agneau (WARDROP, 1960 ; LARGE, 1964).

Si nous admettons qu'il existe une relation entre le temps de présence à l'auge et la quantité ingérée, les chevreaux doubles du lot à bas niveau d'alimentation mangent plus de fourrage vert que les simples. Cette différence de comportement pourrait s'expliquer par un niveau insuffisant de la production laitière journalière des mères de doubles $(630 \mathrm{~g} / \mathrm{j} \pm 56)$ et qui ne représente qu'une hausse de $42 \mathrm{p} .100$ par rapport au niveau des mères de simples $(442 \mathrm{~g} / \mathrm{j} \pm 29)$. En revanche, dans le lot à haut niveau d'alimentation, exceptées 3 observations, l'ingestion de Pangola reste similaire. Ce fait est à relier avec une production laitière des mères de jumeaux suffisante $(1120 \mathrm{~g} / \mathrm{j} \pm 55)$ et qui correspond à une hausse de $58 \mathrm{p} .100$ par rapport à la production des mères de simples $(708 \mathrm{~g} / \mathrm{j} \pm 36)$. Toutefois ceci ne peut expliquer la consommation d'aliment concentré importante des simples du lot BN par rapport à celle des doubles.

Les résultats les plus marquants concernent l'ingestion de l'aliment concentré de sevrage. En effet, les jeunes dont les mères reçoivent de l'aliment concentré en 
ingèrent beaucoup plus tôt et en quantité plus importante que ceux dont les mères ne reçoivent que du fourrage. Cette observation pourrait s'expliquer par un phénomène de transmission du comportement alimentaire par la mère. Elle se ferait alors par imitation, les chevreaux étant fréquemment observés près de leur mère quand elle s'alimente. La transmission de préférences alimentaires a été égalcment mise en évidence chez les Ovins, qu'il s'agisse d'aliments de complémentation tels que des blocs de mélasse-urée (Lobato el al., 1980) ou du blé (LYNCH et al., 1983) ou de préférence de zones de pâturages (KEY \& Mc IVER, 1980). Cet apprentissage ne nécessiterait pas la consommation de l'aliment; seules, les informations visuelles et olfactives seraient suffisantes (LoBato et al., 1980). Cependant, l'aliment concentré des mères et celui des jeunes étant de même composition, la transmission du comportement alimentaire pourrait s'expliquer par l'action d'éléments organoleptiques présents dans le lait consécutifs à la prise d'aliment concentré comme cela a déjà été démontré chez le rat (GaleF \& Henderson, 1972).

Un tel phénomène de transmission peut avoir des implications pratiques très importantes. En effet, il serait possible d'orienter les choix alimentaires par une distribution à la mère, même de peu d'importance, d'un aliment au cours d'une période où le jeune serait susceptible d'acquérir cet apprentissage. Ainsi, cela permettrait facilement et à moindre coût de préparer le jeune ruminant à ingérer de nouveaux aliments et par conséquent d'aborder le sevrage dans des conditions optimales. Cependant, des études systématiques sont nécessaires afin de préciser la période d'apprentissage, les quantités qui doivent être distribuées et les répercussions sur le comportement alimentaire post-sevrage.

Reçu en octobre 1984.

Accepté en mars 1985.

\section{Summary \\ Feeding behaviour of Creole kids kept in loose housing conditions from birth to weaning}

Sucking and feeding behaviour of two groups of Creole kids were studied from birth to weaning (12th week). In the LL group (12 kids) dams were only fed green forage ad libitum (Digitaria decumbens) whereas in the $\mathrm{Hl}$ group (14 kids) they received pellets in addition $(0.5 \mathrm{~kg} / \mathrm{animal} / \mathrm{day})$. In both groups kids had access to the dam forage and from the third week, weaning pellets were offered in a feeding trough only accessible to the young. Observations were made once a week from 6 to $12 \mathrm{~h}$. From the fifth week sucking activity was recorded during 24 hours.

Frequency, duration and attempts of sucking were not significantly different between the two groups (fig. 1). Until the 5 th week this frequency was high (13 suckings $/ \mathrm{kid} / 6 \mathrm{~h}$ ) and then decreased significantly from the 6 th week $(6$ suckings $/ \mathrm{kid} / 6 \mathrm{~h})$. The number of attempts followed the same pattern. Sucking length did not change except in the LL group (26 sec until the 4th week vs $18 \mathrm{sec}$ from the 5th to the 12th week). Sucking activity was almost similar in simple and twin kids. During the 24 -h recording of sucking activity the number of suckings per 6-h period was significantly lower between 0 and $6 \mathrm{~h}$, but did not vary during the remaining day (tabl. 1).

The onset of forage intake occurred nearly at the same time in both groups (3th vs 4th week in HL and LL groups, respectively, fig. 2). On the other hand, pellet consumption took place much earlier in kids whose dams were fed pellets (3th vs 6th wcek in HL and LL groups, respectively).

In conclusion, there is a 5-week period during which the kid closely depends on the mother's milk, irrespective of the mother's feeding level. We also show that kids are taught to feed by the dam and this learning could influence later fecding behaviour.

Key words : creole kid, sucking behaviour, feeding behaviour, feeding learning. 


\section{Références bibliographiques}

Alexandre G., 1983. Production laitière des chèvres créoles : facteurs de variation; influence sur la croissance des jeunes. Thèse de Docteur Ingénieur de l'Ecole nationale supérieure agronomique de Rennes, 114 pages.

Baker R.D., LE DU Y.L.P., Barker J.M., 1976. Milk-fed calves. 1. - The effect of milk intake upon the herbage intake and performance of grazing calves. J. Agric. Sci., 87, 187-196.

Brown T.H., 1964. The early weaning of lambs. J. Agric. Sci., 63, 191-204.

Church D.C., 1976. Digestive Physiology and Nutrition of Ruminants. Volume 1. Digestive physiology. 2nd Edition. Church D.C., Ed., Metropolitan printing Co, 2025 NW Overton, Portland, Oregon U.S.A.

Cognie Y., Houix Y., Logeay B., 1971. Données sur la croissance et la reproduction de la chèvre créole en Guadeloupe. II" Conférence internationale de l'Elevage caprin, Tours, France, S.P.E.O.C., Patre Ed., Paris, p. 345-350.

Doney J.M., Peart J.N., 1976. The effect of sustained lactation on intake of solid food and growth rate of lambs. J. Agric. Sci., 87, 511-518.

Economides S., Papachristoforou Ch., Louca A., 1982. The effects of milk and solid feed intake on the pre and postweaning growth of kids. Proc. Third Intern. Conf. on Goat Production and Disease. January 10-15, Tucson, Arizona, U.S.A., p. 493 (Abst.).

EWBANK R., 1967. Nursing and suckling behaviour amongst Clun Forest ewes and lambs. Anim. Behav., 15, 251-258.

FLetcher I.C., 1971. Relationships between frequency of suckling, lamb growth and postpartum oestrous behaviour in ewes. Anim. Behav., 19, 108-111.

GaleF J.B.G., Henderson P.W., 1972. Mother's milk : a determinant of the feeding preferences of weanling rat pups. J. Comp. Physiol. Psychol., 78, 213-219.

Grhad E.A., Morad H.M., 1977. The development of goat's digestive tract. Symposium on Goat Breeding in Mediterranean Countries. Malaga, Granada, Murcia (Spain), 3-7 Oct.

KEY C., MC IVER R.M., 1980. The effects of maternal influences on sheep : breed differences in grazing, resting and courtship behaviour. Appl. Anim. Ethol., 6, 33-48.

Langlands J.P., 1977. The intake and production of lactating Merino ewes and their lambs grazed at different stocking rates. Aust. J. Agric. Res., 28, 133-142.

LARGE R.V. 1964. The development of the lambs with particular reference to the alimentary tract. Anim. Prod., 6, 169-178.

Le Neindre P., Petit M., 1975. Nombre de tétées et temps de pâturage des veaux dans les troupeaux de vaches allaitantes. Ann. Zootech., 24, 553-558.

Le NeINDRE P., 1984. La relation mère-jeune chez les bovins : Influences de l'environnement social et de la race. Thèse de Doctorat ès Sciences naturelles. Université Rennes, 274 pages.

Lobato J.F.P., Pearce G.R., Beilharz R.G., 1980. Effect of early familiarization with dietary supplements on the subsequent ingestion of molasses-urea blocks by sheep. Appl. Anim. Ethol., 6, 149-161.

Lynch J.J., Keogh R.G., Elwin R.L., Green G.C., Mottershead B.E., 1983. Effects of early experience on the post-weaning acceptance of whole grain wheat by fine-wool Merino lambs. Anim. Prod., 36, 175-183.

Mathieu C.M., Wegat-Litre E., 1961. Mise au point d'une méthode d'alimentation des veaux d'élevage. I. - Détermination de la quantité de lait nécessaire. Ann. Zootech., $19,161-175$.

PENNING P.D., Gibi M.J., 1979. The effect of milk intake on the intake of cut and grazed herbage by lambs. Anim. Prod., 29, 53-67.

Ricordeau G., Boccard R., Denamur R., 1960. Mesure de la production laitière des brebis pendant la période d'allaitement. Ann. Zootech., 9, 97-120.

SIEGEL S., 1956. Nonparametric statistics for the behavioral sciences. Mc Graw-Hill, New York, 312 pages.

SOMmERVILLE S.H., Lowman B.G., 1979. Observations on the behaviour of bcef cows suckling Charolais cross calves. Appl. Anim. Ethol., 5, 369-373.

Spedding C.R.M.. Charlet G., 1967. Le sevrage précoce des agneaux. World. Rev. Anim. Prod., 3, 97-109.

WARDROP I.D., 1960. The post-natal growth of the visceral organ of the lambs. II. - The effect of the diet on growth rate with particular reference to the parts of the alimentary tract. J. Agric. Sci., 55, 127-131. 